\title{
Membrane sorption property effects on transmembrane permeation
}

\author{
MIN JingChun* \& WANG LiNing \\ School of Aerospace, Tsinghua University, Beijing 100084, China
}

Received January 19, 2011; accepted May 11, 2011

\begin{abstract}
A theoretical study was carried out into membrane transport phenomena. Formulae for calculating the membrane transport resistance and transmembrane mass flux were given, variations in membrane resistance and moisture flux with the membrane sorption constant $(C)$ under various humidity conditions were analyzed, and the value of $C$ corresponding to the minimum membrane resistance or the maximum moisture flux was obtained. The results show that the membrane resistance and moisture flux relate not only to $C$ but also to the relative humidities on both sides of the membrane. As $C$ increases, membrane resistance initially decreases but then increases, i.e., a minimum occurs, while the moisture flux first decreases and then increases, i.e., a maximum occurs. The membrane resistance and moisture flux reach their extrema at the same value of $C$, which is determined by the relative humidities on both sides of the membrane. To reduce the membrane resistance, the value of $C$ should be chosen based on the humidity conditions.
\end{abstract}

membrane transport, membrane resistance, sorption, mass transfer

Citation: Min J C, Wang L N. Membrane sorption property effects on transmembrane permeation. Chinese Sci Bull, 2011, 56: 2394-2399, doi: $10.1007 / \mathrm{s} 11434-011-4562-4$

Membrane separation is an emerging separation technology, widely used in various fields because of its low energy consumption, high efficiency, and other advantages [1]. Although membrane separation technology has been successfully applied to large-scale liquid phase separations, its application to gas separation is a relatively new area. Applications of membrane separations in the gas phase have high potential value, with examples including the adjustment of natural gas dew point, energy and moisture recoveries in a membrane-based total heat exchanger, amongst others [2]. There are two types of membrane material used for gas separation: inorganic and organic membranes, with the latter representing the current state of development for membrane gas separations. However, organic membranes may have unstable performance under certain conditions, such as in the presence of corrosive chemicals or at high temperature and/or pressure, while an inorganic membrane may effectively overcome these problems and simultaneously yield higher permeability and better selectivity [3].

*Corresponding author (email: minjc@tsinghua.edu.cn)
There are currently two theoretical models for mass transfer in membrane gas separations: the pore flow model and the solution-diffusion model. The pore flow model mainly applies to mass transfer in a porous membrane and the relevant mass transfer mechanism relates to the Knudsen number, while the solution-diffusion model is currently the most mature model used for membrane gas separations [4]. The latter assumes that the transmembrane permeation process comprises three steps including adsorption, diffusion, and desorption: the gas first adsorbs at the membrane surface on the high concentration side because of physical or chemical effects, then diffuses from the high to the low concentration side, driven by the concentration potential difference, and eventually desorbs from the membrane surface on the low concentration side.

In the solution-diffusion model, the sorption characteristics of the membrane surface have important influences on the membrane transport process. The sorption property of the membrane can be described using sorption isotherms, with one popular sorption isotherm equation widely used in gas separations having the form [5]: 


$$
\theta=\frac{w_{\max }}{1-C+C /\left[P_{v} / P_{s}(T)\right]},
$$

where $P_{v}$ expresses the adsorbate vapor partial pressure, $P_{s}(T)$ denotes the saturated vapor pressure corresponding to the system temperature, $T, w_{\max }$ is the maximum vapor content of the membrane corresponding to $p_{v}=p_{s}(T)$ and $C$ is the membrane sorption constant. When eq. (1) is applied to the adsorption of water vapor from moist air, it can be written as $[6,7]$

$$
\theta=\frac{w_{\max }}{1-C+C / \phi}
$$

where $\phi$ expresses the air relative humidity.

$C$ is a variable that represents membrane characteristics; it determines the shape of the sorption curve, as shown in Figure 1. $C=1$ indicates that the sorption quantity of water vapor is linearly related to the relative humidity of moist air, which corresponds to Henry sorption. $C<1$ yields an upward convex curve, which corresponds to Langmuir sorption. $C>1$ generates a downward convex curve, which applies to a variety of desiccants [8-10]. Min et al. [10] experimentally investigated the sorption properties of different membranes, including PVDF (polyvinylidene fluoride), PES (polyether sulfone) and cellulose and reported that their sorption constants as $10.26,3.12$ and 6.28, respectively.

$C$ has a substantial influence on the membrane transport process. Simonson and Besant [6,7] studied the latent heat efficiencies corresponding to three different values of $C$. Niu and Zhang [8] analyzed the effects of the entering air humidity and maximum sorption capacity on the effectiveness of a membrane-based total heat exchanger for three different $C$ values. They also calculated the variations of the membrane diffusion resistance coefficient with the air humidity for the same $C$ values. Min and $\mathrm{Su}$ [11] presented the continuous changes in membrane resistance with $C$ for a membrane-based total heat exchanger and found that as $C$ increases, the membrane resistance initially decreases and then begins to increase, with the minimum resistance occur-

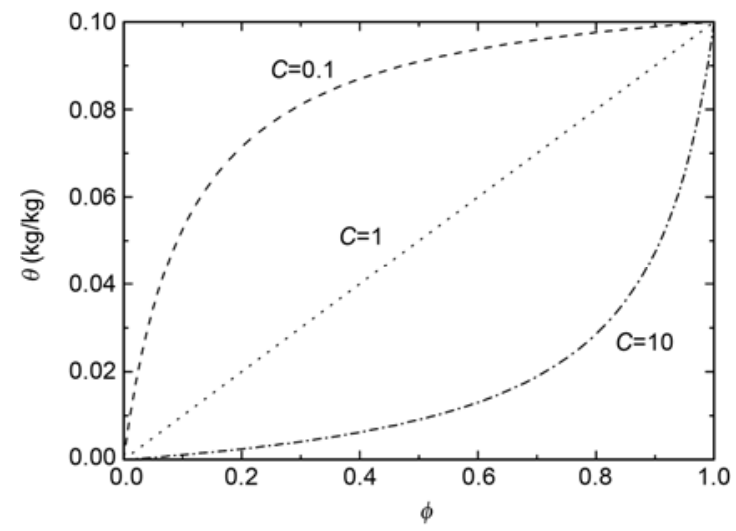

Figure 1 Sorption isotherms of membranes. ring in the vicinity of but not at the point $C=1$. Min et al. [12] investigated the effects of membrane sorption properties on the membrane transport process by combining experimental measurements and numerical simulations. They also reported that the minimum moisture resistance took place at a value of $C$ close but unequal to unity. These studies motivated the present authors to pursue the current research. The purpose of this study is to investigate the effect of membrane sorption properties on the membrane transport process by adopting the most fundamental physical model of membrane transport. Membrane resistance is used to characterize the transmembrane permeation, as in Min and Su [11], Min et al. [10,12], and many other publications on membrane transport [13-19]. Transmembrane transfer of water vapor in moist air is used as an example but the theory and methodology can apply equally well to the other cases. Relationships between membrane resistance, moisture flux and $C$ are determined and the effects of $C$ on membrane resistance and moisture flux are analyzed at various air humidities.

\section{Theoretical model}

Consider the process of one-dimensional moisture transfer through a membrane, as shown in Figure 2, and assume the system temperature to be $T$, the air relative humidities on the left and right sides of the membrane to be $\phi_{1}$ and $\phi_{2}$, respectively, and with $\phi_{1}>\phi_{2}$. According to the solutiondiffusion theory, the water vapor will transfer from the left to the right side of the membrane because of the concentration gradient, causing dehumidification of moist air on the left side of the membrane. To highlight the membrane transport process itself, the convective mass transfer resistances on both sides of the membrane are neglected, i.e., the air humidities on both sides of the membrane are considered to be constant (no concentration boundary layers exist) [20].

According to Fick's law, the mass flux through the membrane can be represented by

$$
J=\frac{D_{w m}}{\delta}\left(\theta_{1}-\theta_{2}\right)
$$

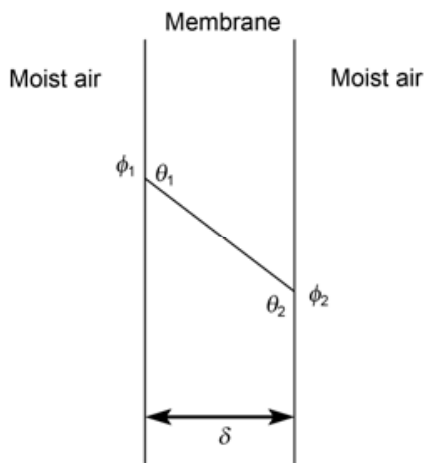

Figure 2 Moisture transfer across a membrane. 
where $D_{w m}$ is the moisture diffusivity in the membrane $\left(\mathrm{kg} \mathrm{m}^{-1} \mathrm{~s}^{-1}\right)$ and is assumed here to be constant, $\delta$ is the membrane thickness $(\mathrm{m})$, and $\theta_{1}$ and $\theta_{2}$ are the moisture uptakes of the membrane surfaces on the two sides of the membrane $\left(\mathrm{kg} \mathrm{kg}^{-1}\right)$, with $\theta$ and the air relative humidity $\phi$ satisfying eq. (1(a)). Correspondingly, the moisture transfer resistance through membrane can be expressed as

$$
R=\frac{w_{1}-w_{2}}{J}=\frac{\delta}{D_{w m}} \frac{w_{1}-w_{2}}{\theta_{1}-\theta_{2}},
$$

where $w_{1}$ and $w_{2}$ are the air humidity ratios on the two sides of the membrane. Substituting eq. (1(a)) into eq. (2) yields

$$
J=\frac{D_{w m} w_{\max }}{\delta}\left(\frac{1}{1-C+C / \phi_{1}}-\frac{1}{1-C+C / \phi_{2}}\right) .
$$

At atmospheric pressure, the air temperature, humidity ratio, and relative humidity approximately meet the relation [8]:

$$
\frac{\phi}{w}=\frac{\mathrm{e}^{5294 / T}}{10^{6}},
$$

$T$ is the absolute temperature in K. eq. (5) is valid for the temperatures range from 273.15 to $323.15 \mathrm{~K}$. Substituting eqs. (1(a)) and (5) into eq. (3) gives

$$
R=\frac{10^{6} \delta}{D_{w m} w_{\max } \mathrm{e}^{5294 / T}} \frac{\phi_{1}-\phi_{2}}{\frac{1}{1-C+C / \phi}-\frac{1}{1-C+C / \phi_{2}}},
$$

Eqs. (4) and (6) can be expressed as

$$
\begin{gathered}
J=\frac{D_{w m} w_{\max }}{\delta} \frac{C \Delta \phi}{\left[(1-C) \phi_{\text {ave }}+C\right]^{2}-[(1-C) \Delta \phi / 2]^{2}}, \\
R=\frac{10^{6} \delta}{D_{w m} w_{\max } e^{5294 / T}} \frac{1}{C}\left\{\left[(1-C) \phi_{\text {ave }}+C\right]^{2}-[(1-C) \Delta \phi / 2]^{2}\right\},
\end{gathered}
$$

where

$$
\phi_{\text {ave }}=\frac{\phi_{1}+\phi_{2}}{2}, \quad \Delta \phi=\phi_{1}-\phi_{2},
$$

$\phi_{\text {ave }}$ denotes the average relative humidity of the system and reflects the humidity level of the system, while $\Delta \phi$ denotes the relative humidity difference between the two sides of the membrane and reflects the magnitude of the mass transfer driving potential between the two sides of the membrane.

\section{Results and discussion}

Permeation of water vapor in moist air across a membrane can be used as an example to analyze the effects of the sorption parameter $C$ on the membrane resistance and moisture flux. The calculations use $T=300 \mathrm{~K}, D_{w m} / \delta=$
$0.0225 \mathrm{~kg} \mathrm{~m}^{-2} \mathrm{~s}^{-1}$, and $w_{\max }=0.1 \mathrm{~kg} \mathrm{~kg}^{-1}$.

It can be found from eqs. (6) and (8) that the membrane resistance is related not only to the membrane sorption constant $C$ but also to the relative humidities on both sides of the membrane. The variations in membrane resistance $R$ with $C$ were first calculated for different relative humidity differences between the two sides of the membrane, $\Delta \phi=0.1$, 0.3 and 0.5 , at various average relative humidities, and the results are shown in Figure 3(a)-3(c), corresponding to the cases $\phi_{\text {ave }}=0.3,0.5$ and 0.7 , respectively. When $C$ is varied from 0.1 to 10 , the membrane resistance initially decreases and then increases, i.e., a minimum occurs. The value of $C$ corresponding to the minimum membrane resistance relates to the average humidity: for $\phi_{\mathrm{ave}}=0.3$, the minimum appears in the range of $C<1$; for $\phi_{\mathrm{ave}}=0.5$, the minimum occurs at $C=1$; for $\phi_{\mathrm{ave}}=0.7$, the minimum takes place in the range of $C>1$. Also, for $C=1$, the three curves coincide at the same average humidity. This can be understood using eq. (8), that is, for $C=1$, the membrane resistance is independent of the relative humidities on both sides of the membrane and is related only to $C$.

Comparison of Figure 3(a)-3(c) reveals that for a given relative humidity difference, if $C<1$, a low average humidity will lead to a small membrane resistance, while if $C>1$, a low average humidity will lead to a large membrane resistance. This can be explained as follows. It is known from Figure 1 that, for $C<1$, a low humidity corresponds to a large $\mathrm{d} \theta / \mathrm{d} \phi$. eq. (3) indicates that the membrane resistance is inversely proportional to $\mathrm{d} \theta / \mathrm{d} w=(\mathrm{d} \theta / \mathrm{d} \phi)(\mathrm{d} \phi / \mathrm{d} w)$, while eq. (5) shows that for a fixed temperature $\mathrm{d} \phi / \mathrm{d} w$ remains constant. Therefore, $R$ is inversely proportional to $\mathrm{d} \theta / \mathrm{d} \phi$ so a low humidity will lead to a small membrane resistance. Similarly, for $C>1$, a low humidity corresponds to a small $\mathrm{d} \theta / \mathrm{d} \phi$, leading to a large membrane resistance.

To obtain the value of $C$ corresponding to the minimum membrane resistance, differentiating eq. (8) with regard to $C$ and letting $\mathrm{d} R / \mathrm{d} C=0$ yields

$$
C=\sqrt{\frac{\left(\phi_{\text {ave }}\right)^{2}-(\Delta \phi / 2)^{2}}{\left(1-\phi_{\text {ave }}\right)^{2}-(\Delta \phi / 2)^{2}}} .
$$

The above equation shows that the value of $C$ corresponding to the minimum membrane resistance is related not only to the average humidity but also to the humidity difference between the two sides of the membrane. In combination with Figure 3, the values of $C$ corresponding to the minimum membrane resistances were calculated using eq. (10) and the results are presented in Figure 4. The figure shows that the value of $C$ corresponding to the minimum membrane resistance increases with increasing average humidity $\left(\phi_{\text {ave }}\right)$. For $\phi_{\text {ave }}=0.5$, the value of $C$ corresponding to the minimum membrane resistance is independent of the relative humidities on both sides of the membrane and is always equal to unity; for $\phi_{\text {ave }} \neq 0.5$, however, the value of $C$ 

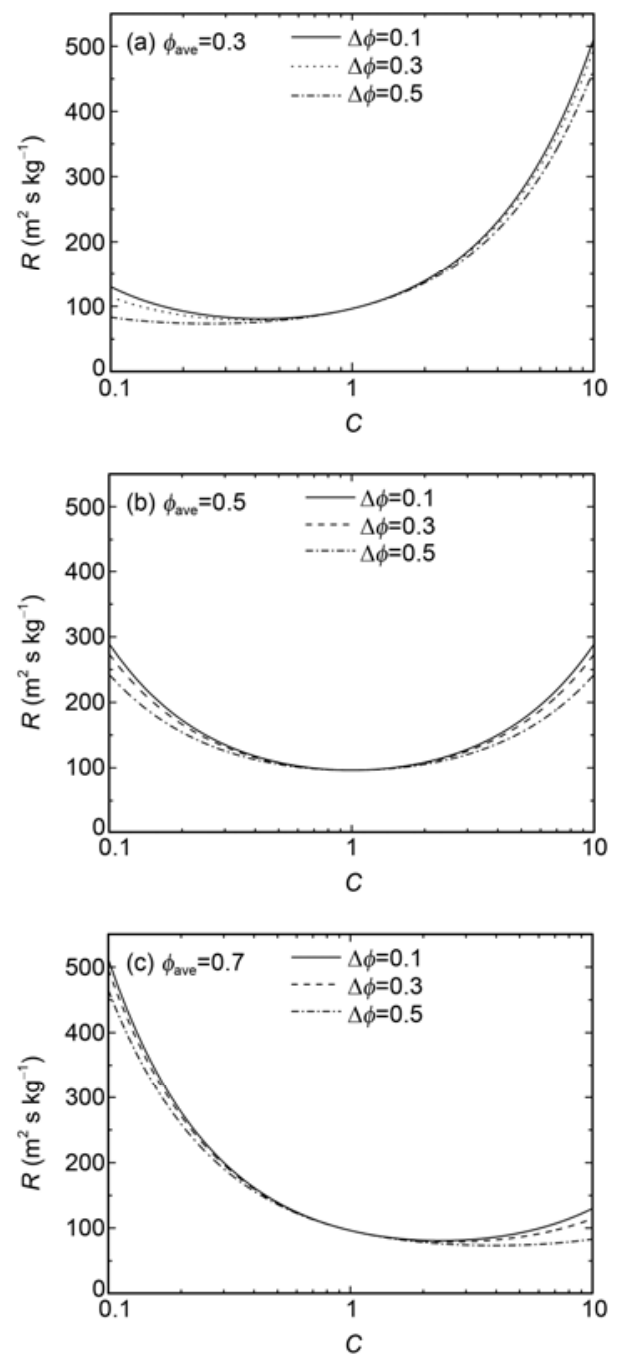

Figure 3 Variations in membrane resistance with $C$ under various humidity conditions. (a) $\phi_{\mathrm{ave}}=0.3$, (b) $\phi_{\mathrm{ave}}=0.5$, (c) $\phi_{\mathrm{ave}}=0.7$.

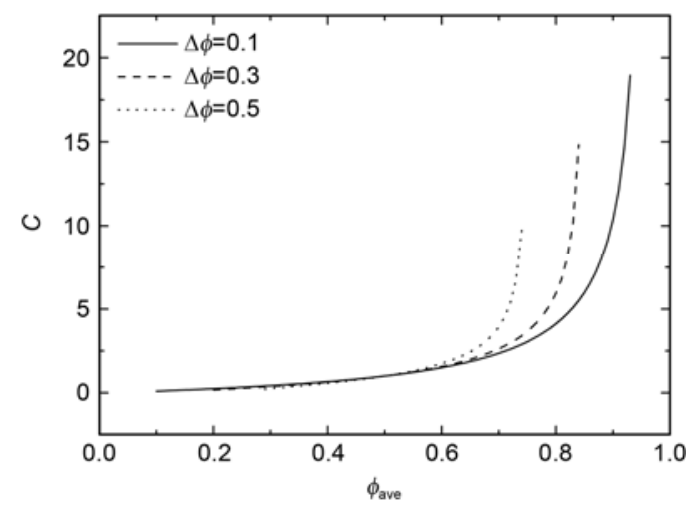

Figure 4 Variations in the value of $C$ corresponding to the minimum membrane resistance with humidity.

corresponding to the minimum membrane resistance is related to the relative humidities on both sides of the membrane. For $\phi_{\mathrm{ave}}<0.5$, the value of $C$ decreases with increasing humidity difference, while for $\phi_{\mathrm{ave}}>0.5$, the value of $C$ in- creases with increasing humidity difference. These results may provide guidance for selection of the membrane materials.

The variations in moisture flux with $C$ corresponding to Figure 3 are depicted in Figure 5. For a fixed average humidity, a large humidity difference between the two sides of the membrane will lead to a large moisture flux, and vice versa. The reason for this is that, on the one hand, a large humidity difference creates a large driving potential for mass transfer, while on the other hand, according to the earlier analysis of the membrane resistance, when the humidity difference is increased, the membrane resistance decreases. These two factors, together, lead to the result that a large humidity difference causes a large moisture flux. Further, when $C$ is increased, the moisture flux initially increases and then decreases. This is because for a fixed humidity
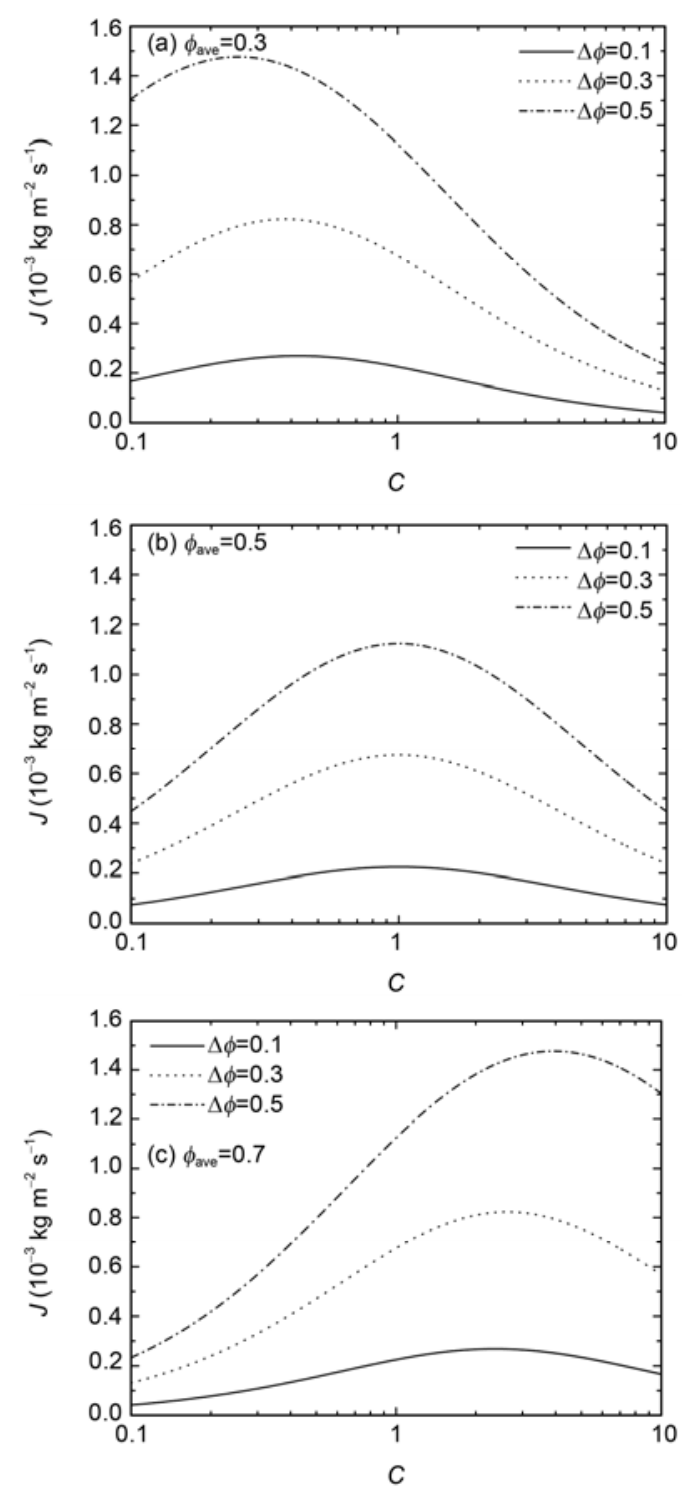

Figure 5 Variations of moisture flux with $C$ under various humidity conditions. (a) $\phi_{\mathrm{ave}}=0.3$, (b) $\phi_{\mathrm{ave}}=0.5$, (c) $\phi_{\mathrm{ave}}=0.7$. 
difference, i.e., a fixed driving force, when $C$ is increased, the membrane resistance first increases and then decreases, so the moisture flux first increases and then decreases. Also, it can be seen from Figure 5 that the maximum moisture flux occurs at a certain value of $C$. Differentiating eq. (7) with respect to $C$ and letting $\mathrm{d} J / \mathrm{d} C=0$ will equally yield eq. (10). This suggests that the maximum moisture flux and the minimum membrane resistance occur at the same value of $C$.

Further, it can be seen from Figure 3 that as the humidity difference between the two sides of the membrane decreases, the membrane resistance increases. As the humidity difference approaches zero, the moisture flux approaches zero, while the membrane resistance is unequal to zero and the membrane resistance can be expressed as (in this case $\left.\phi_{\mathrm{ave}}=\phi_{1}=\phi_{2}=\phi\right)$ :

$$
R=\frac{10^{6} \delta}{D_{w m} w_{\max } \mathrm{e}^{5294 / T}} \frac{1}{C}[(1-C) \phi+C]^{2} .
$$

The varying curves for membrane resistance with $C$ under different humilities, calculated using eq. (11), are presented in Figure 6. Similar to the results shown in Figure 3, as $C$ increases, the membrane resistance initially increases and then decreases, i.e., a minimum occurs. With an increase in humidity, the value of $C$ corresponding to the minimum membrane resistance increases. Actually, when the humidity difference between the two sides of the membranes approaches zero, eq. (10) becomes

$$
C=\frac{\phi}{1-\phi} \text {. }
$$

The variation of $C$ with the relative humidity in this case is demonstrated in Figure 7, which shows that with an increase in humidity, the value of $C$ corresponding to the minimum membrane resistance increases. This result is certainly consistent with those shown in Figure 4.

\section{Conclusions}

(1) A theoretical study was carried out to investigate the

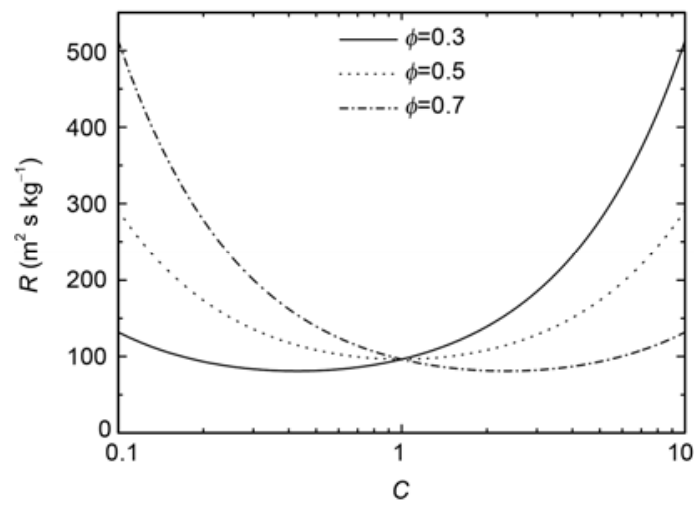

Figure 6 Variations in membrane resistance with $C$ when the humidity difference approaches zero.

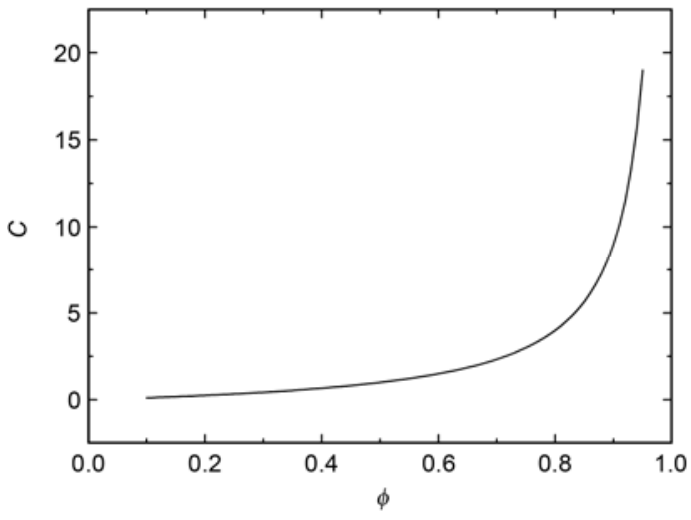

Figure 7 Variations in $C$ corresponding to the minimum membrane resistance with humidity when the humidity difference approaches zero.

moisture transfer process across a membrane and equations for calculating the moisture transfer resistance and moisture flux through membrane were obtained. The equations support the conclusion that the membrane resistance and moisture flux are related not only to the membrane sorption constant, $C$, but also to the relative humidities on both sides of the membrane.

(2) The variations in membrane resistance with $C$ under various humidity conditions were investigated and the results show that as $C$ increases the membrane resistance initially decreases and then increases, i.e., a minimum occurs. The value of $C$ corresponding to the minimum membrane resistance is related to the average humidity: when the average relative humidity equals 0.5 , the minimum appears at the point $C=1$; when the average relative humidity is less than 0.5 , the minimum appears in the range $C<1$; and when the average relative humidity exceeds 0.5 , the minimum appears in the range $C>1$. Furthermore, for a fixed average humidity, the smaller the relative humidity difference between the two sides of the membrane, the larger the membrane resistance.

(3) The variations of moisture flux with $C$ at various air humidities were investigated and the results show that as $C$ increases the moisture flux initially increases and then decreases, i.e., a maximum occurs. The maximum moisture flux occurs at the value of $C$ at which the minimum membrane resistance takes place.

(4) The values of $C$ corresponding to the minimum membrane resistance or the maximum moisture flux were calculated and the results show that they relate to both the average relative humidity and the relative humidity difference between the two sides of the membrane. To reduce the membrane resistance, the value of $C$ should be chosen in light of the humidity conditions, providing a guide for the manufacture and use of some type of membrane.

This work was supported by the National Natural Science Foundation of China (50576040). 
1 Wang Z S, Gu Z L, Feng S Y, et al. Applications of membrane distillation technology in energy transformation process-basis and prospect. Chinese Sci Bull, 2009, 54: 2766-2780

$2 \mathrm{Hu}$ T, Min J C, Song Y Z. Analysis of the effects of mass transfer on heat transfer in the progress of moisture exchanger across a membrane. Chinese Sci Bull, 2010, 55: 1221-1225

3 Merritt A R. The design of high flux nanoporous carbon membranes and their application in small gas molecule separation. Doctoral Disseration. Ann Arbor: The Pennsylvania State University, 2007

4 Bitter J G A. Transport Mechanisms in Membrane Separation Processes. Plenum Press: New York, 1991

5 Zheng Z, Worek W M. Numerical simulation of combined heat and mass transfer process in a rotary dehumidifier. Numer Heat Transfer, Part A, 1993, 23: 211-232

6 Simonson C J, Besant R W. Energy wheel effectiveness, Part 1 - development of dimensionless groups. Int J Heat Mass Transfer, 1999, 42: 2161-2170

7 Simonson C J, Besant R W. Energy wheel effectiveness, Part 2 correlations. Int J Heat Mass Transfer, 1999, 42: 2171-2185

8 Niu J L, Zhang L. Membrane-based enthalpy exchanger: material considerations and clarification of moisture resistance. J Mem Sci, 2001, 189: 179-191

9 Kadylak D, Cave P, Merida W. Effectiveness correlations for heat and mass transfer in membrane humidifiers. Int J Heat Mass Transfer, 2009, 52: 1504-1509

10 Min J C, Hu T, Liu X W. Evaluation of moisture diffusivities in various membranes. J Mem Sci, 2010, 357: 185-191

11 Min J C, Su M. Performance analysis of a membrane-based enthalpy exchanger: Effects of the membrane properties on the exchanger per- formance. J Mem Sci, 2010, 348: 376-382

12 Min J C, Hu T, Song Y Z. Experimental and numerical investigations of moisture permeation through membranes. J Mem Sci, 2011, 367: 174-181

13 Hu T, Min J C, Song Y Z. Modeling and analysis of dynamic adsorption during gas transport through a membrane. J Mem Sci, 2009, 39: 204-208

14 Gibson P, Kendrick C, Rivin D, et al. An automated water vapor diffusion test method for fabrics, laminates, and films. J Coat Fabrics, 1995, 24: 322-345

15 Majsztrik P W, Satterfield M B, Bocarsly A B, et al. Water sorption, desorption and transport in Nafion membranes. J Mem Sci, 2007, 301: 93-106

16 Liu L, Chen Y, Li S G, et al. The Effect of a support layer on the permeability of water vapor in asymmetric composite membranes. Sep Sci Technol, 2001, 36: 3701-3720

17 Zhang L Z. Fabrication of a lithium chloride solution based composite supported liquid membrane and its moisture permeation analysis. J Mem Sci, 2006, 276: 91-100

18 Larson M D, Simonson C J, Besant R W, et al. The elastic and moisture transfer properties of polyethylene and polypropylene membranes for use in liquid-to-air energy exchangers. J Mem Sci, 2007, 302: 136-149

19 Gibson P, Schreuder-Gibson H, Rivin D. Transport properties of porous membranes based on electrospun nanofibers. Colloids Surf, A: Physicochem Engin Aspects, 2001,187-188: 469-481

20 Wang L N, Min J C. Studies of the process of moisture exchange across membrane using irreversible thermodynamics. Chinese Sci Bull, 2011, 56, doi: 10.1007/s11434-010-4238

Open Access This article is distributed under the terms of the Creative Commons Attribution License which permits any use, distribution, and reproduction in any medium, provided the original author(s) and source are credited. 